\title{
Mirtazapine versus venlafaxine in hospitalized severely depressed patients with melancholic features
}

Julien Daniel Guelfi*, Marc Ansseau†, L. Timmerman‡, S. Kørsgaard§, The MirtazapineVenlafaxine Study Group

* Hôpital Paul Brousse, Villejuif, France;

†C.H.U. Service de Psychiatrie, Liège, Belgium;

$\neq$ Albert Schweitzer Ziekenhuis, Dordrecht, The Netherlands;

SDepartment of Psychiatry, Esbjerg Hospital, Esbjerg, Denmark

KEYWORDS: Depressive disorder, Cyclohexanols, Mianserin, Psychiatric status rating scales

\section{ABSTRACT}

The aim of this multicenter, randomized, double-blind, 8-week study was to compare the antidepressant efficacy and tolerability of mirtazapine and venlafaxine in the treatment of hospitalized patients with DSM-IV diagnosis of severe depressive episode with melancholic features. Patients with a baseline score of $>$ or $=25$ on the 17-item Hamilton Rating Scale for Depression (HAM-D-17) were randomly assigned to receive treatment with either mirtazapine $(\mathrm{N}=78,15-60 \mathrm{mg} /$ day $)$ or venlafaxine $(\mathrm{N}=79,75-375 \mathrm{mg} /$ day, twice a day) in a rapid uptitration schedule. Efficacy was assessed with the Montgomery-Asberg Depression Rating Scale (MADRS), HAM-D-17, and Clinical Global Impression scale, and quality of life was assessed with the Quality of Life, Enjoyment, and Satisfaction Questionnaire and Quality of Life in Depression Scale. Tolerability was assessed with the Utvalg for Kliniske Undersogelser (UKU) side effect scale and by reporting adverse events. Both drugs were effective in reducing overall symptoms of depression, showing substantial reductions in group mean MADRS scores (-20.1 for mirtazapine and -17.5 for venlafaxine) and HAM-D-17 scores (-17.1 for mirtazapine and -14.6 for venlafaxine) at the end of the treatment. Although not statistically significant, at all assessment times higher percentages of patients treated with mirtazapine were classified as responders ( $>$ or $=50 \%$ reduction) on the HAM-D (at endpoint, $62 \%$ vs. $52 \%$ ) and MADRS (at endpoint: $64 \%$ vs. $58 \%$ ). Likewise were the percentages of remitters (HAM-D score $<$ or $=7$; MADRS score $<$ or $=12$ ) also higher in the mirtazapine group. A statistically significant difference favoring mirtazapine was found on the HAM-D Sleep Disturbance factor at all assessment points $(\mathrm{p}<$ or $=0.03$ ). Both treatments were well tolerated. Although slightly more subjects treated with mirtazapine reported at least one adverse event, a statistically significantly higher percentage of patients treated with venlafaxine (15.3\%) than mirtazapine $(5.1 \%)$ dropped out because of adverse events $(p=0.037)$. Quality of life improved in both treatment groups. In this study, treatment with mirtazapine resulted in a trend toward more responders and remitters than treatment with venlafaxine and in significantly fewer dropouts as a result of adverse events. 
The term melancholia denotes a variety of conditions, and it has most often been used as a term for depression. Its use was readopted in DSM-III as an operationally defined subtype of a major depressive disorder. ${ }^{1}$ The usage continued in DSM-III-R ${ }^{2}$ and DSMIV, ${ }^{3}$ which currently uses the "melancholic features" specifier for the major depressive episode or bipolar I or II major depressive episode. The essential features are anhedonia and loss of reactivity to usually pleasurable stimuli. Other common features are diminished or absent mood reactivity, psychomotor changes observable by others, excessive guilt, anorexia or weight loss, early morning awakenings, and a depressed mood that is worse in the morning. Several studies suggested that tricyclic antidepressants (TCAs), and in particular the dual-acting clomipramine, are superior to selective serotonin reuptake inhibitors (SSRIs) in treating melancholic depression (for a review, see Amsterdam ${ }^{1}$ ). However, TCAs are often not as well tolerated as the SSRIs and the newer agents. Venlafaxine, a dual-acting serotonin and noradrenaline reuptake inhibitor (SNRI), has been demonstrated more effective than placebo ${ }^{2}$ and fluoxetine ${ }^{3}$ in treating hospitalized patients with melancholic depression, and this also suggests the superiority of dualacting antidepressants in these patients. The efficacy of mirtazapine (Remeron, N.V. Organon, Oss, The Netherlands), a dual-acting noradrenergic and specific serotonergic antidepressant (NaSSA), is established in placebo-controlled studies, ${ }^{4}$ and it demonstrated superiority over fluoxetine ${ }^{5}$, citalopram, ${ }^{6}$ and paroxetine ${ }^{7}$ in patients who were moderately to severely depressed. The aim of this study was to compare the efficacy and tolerability of mirtazapine and venlafaxine in hospitalized patients who were severely depressed with melancholic features, in a randomized, double-blind, 8-week study.

\section{Methods}

This study was a multicenter, randomized, double-blind comparison of mirtazapine and venlafaxine, performed in 23 centers in France, 4 centers in Belgium, 2 centers in Denmark, and 4 centers in The Netherlands in the period between January 1997 and July 1999. Patients meeting inclusion and exclusion criteria were recruited from psychiatric inpatient populations. The ethics committee of each participating center approved the study. Each participating patient gave written informed consent before starting any study-related activity. The study was conducted in compliance with the current revision of the Declaration of Helsinki, International Conference for Harmonization guidelines, good clinical practice, and current regulatory regulations in the countries where the study was conducted.

Male and female hospitalized patients between 18 and 65 years old who fulfilled DSM-IV criteria for a severe depressive episode (296.23 or 296.33) with melancholic features according to the DSM-IV checklist, had a total score of $\geq 25$ on the 17 -item Hamilton Rating Scale for Depression (HAM-D-17) 8 at the start and end of the placebo washout period, and who were willing to give informed consent in writing were eligible for participation in this study.

Reasons for exclusion included a current depressive episode of more than 12 months' duration, more than two previous episodes of major depression that did not respond to adequate antidepressant therapy, lack of response to at least two antidepressant therapies given for at least 6 weeks during the current episode, a reduction of $\geq 25 \%$ in the HAM-D-17 score during the placebo washout period, a major suicide risk, a history of or presence of a bipolar disorder, 
depressive disorder not otherwise specified, depression with psychotic features, depression with atypical features, depression with postpartum onset, depression with catatonic features, or organic mental disorders. Subjects with a present condition of anxiety disorders (according to DSM-IV), eating disorders (specifically, anorexia nervosa and bulimia nervosa), epilepsy or a history of a seizure disorder or of a treatment with anticonvulsant medication for epilepsy or seizures, alcohol intoxication or substance abuse (according to DSM-IV), schizophrenia, or other psychotic disorders were excluded. Female patients were also excluded if they were pregnant, lactating, or of childbearing potential and not taking adequate contraceptive measures. Presence of any clinically meaningful nonstable physical disease or abnormal findings on physical examination or laboratory testing were reasons for exclusion. The following treatments must have been stopped within the indicated intervals before the start of active study medication: electroconvulsive therapy (3 months, and not for the actual episode), neuroleptics and depot neuroleptics for antipsychotic purpose (2 months) or neuroleptics as anxiolytics (4 days), fluoxetine (4 weeks), benzodiazepines (patients receiving oxazepam with a minimum dose of 50 $\mathrm{mg} /$ day, without a washout period), monoamine oxidase inhibitors (2 weeks), moclobemide (3 days), mirtazapine (current episode), venlafaxine (current episode), and other psychotropic drugs ( 1 week).

Subjects were not permitted to use antihypertensive medication such as guanethidine, guanoxan, clonidine, prazosin, or $\alpha$-methyldopa. Any psychotropic medications other than the trial medication were not allowed in the protocol; sedative drugs (including sedative antihistaminics), anticholinergic drugs, and sympathomimetic drugs (including epinephrine) were not allowed, nor was concurrent psychotherapy for the treatment of depression or formal psychotherapy. The following concomitant medications were allowed: medication for the mild physical illness other than defined in the exclusion criteria; in case of sleeping problems, a maximum of $20 \mathrm{mg}$ of zolpidem; and, in case anxiety symptoms were a reason for discontinuation, short-acting benzodiazepines (e.g., oxazepam, maximally $50 \mathrm{mg} /$ day, not more than three times per week, and not on the day before an assessment).

After a 3- to 7-day placebo washout period, patients were randomized to receive treatment with either mirtazapine or venlafaxine orally for 8 weeks, prepared as indistinguishable capsules, according to a centrally prepared randomization list. Packaging was performed by use of a double-dummy technique. The dosing regimen for both drugs was in line with the standard labeling. To match differences in morning and evening administration, subjects treated with mirtazapine were to take a placebo capsule in the morning. The dose of mirtazapine was 15 $\mathrm{mg} /$ day on days 1 and 2, $30 \mathrm{mg}$ /day on days 3 through 5, and $45 \mathrm{mg} /$ day on days 6 through 8 . From day 9 onward, the daily dose could be increased to $60 \mathrm{~g} /$ day, depending on the clinical response. The dose of venlafaxine was $75 \mathrm{mg} /$ day, twice a day, during days 1 and 2; $150 \mathrm{mg} /$ day during days 3 through 5; and $225 \mathrm{mg}$ daily from day 6 onward, until trial completion. This dose could be increased to 300 or $375 \mathrm{mg}$ (depending on the clinical response) daily from day 9 to day 56.

Assessments were performed at screening, at baseline (day 0), and at weeks 1, 2, 4, 6, and 8 of active treatment (or on premature withdrawal). Efficacy was assessed with the MontgomeryÅsberg Depression Rating Scale (MADRS), ${ }^{9}$ HAM-D-17, and Clinical Global Impression (CGI) scales (Severity of Illness and Global Improvement). ${ }^{10}$ Patient-rated Quality of Life, Enjoyment, 
and Satisfaction Questionnaires (QLESQ) ${ }^{11}$ and Quality of Life in Depression Scales (QLDS) ${ }^{12}$ were used in local languages and completed at weeks 2 , 4, and 8 . Secondary efficacy parameters, such as percentages of HAM-D-17 and MADRS responders, were defined as the percentage of patients with a $\geq 50 \%$ decrease from baseline scores. HAM-D-17 and MADRS remitters were defined as patients with a total score of $\leq 7$ and a total score of $\leq 12$, respectively. Tolerability was assessed by the Utvalg for Kliniske Undersogelser (UKU) side effect scale ${ }^{13}$ and by the registration of adverse events at baseline and at weeks 1, 2, 4, 6, and 8. Vital signs were measured at the same assessment points, as were laboratory variables at baseline and at the last visit. All adverse events were coded by using the dictionary terms from the World Health Organization adverse reaction terminology (1997 version). ${ }^{14}$

All investigators were trained in using the rating scales 1 month before the start of the trial and once during the trial to achieve a maximum consistency between and within raters. All subjects were to be instructed by the investigators on the use of the Quality of Life questionnaire. Sample-size calculations were based on the assumption that at least 65 patients per group (minimal size of the intent-to-treat [ITT] group) would complete 4 weeks of treatment. With this sample size, the trial would have a $90 \%$ power to detect a difference of $35 \%$ versus $10 \%$ (twosided testing at $\mathrm{p}=0.05$ ) and a power of $80 \%$ to detect a significance level of $5 \%$.

Efficacy analyses were based on the ITT patient sample, thus including all randomly assigned subjects who received at least one dose of study medication and had at least one postbaseline efficacy assessment, by using the last observation carried forward method. Changes from baseline in the total MADRS and HAM-D-17 scores, HAMD factor scores, CGI Severity of Illness, QLESQ, and QLDS scores were statistically analyzed by means of an analysis of variance (ANOVA). The estimates of treatment difference and corresponding two-sided 95\% confidence intervals were based on the additive two-way ANOVA with factors treatment and country. Secondary efficacy variables, such as percentages of HAM-D-17 and MADRS responders (patients with a $\geq 50 \%$ decrease from baseline scores), HAM-D-17 remitters (total score of $\leq 7$ ), and MADRS remitters (total score of $\leq 12$ ) were analyzed with the Cochran-Mantel-Haenszel test, 15 adjusting for country. The CGI Global Improvement scores were analyzed by means of the Wilcoxon test, adjusting for country, but for the communication of results, an ANOVA model was used. The primary time point for treatment comparison was the endpoint assessment, with week- 4 assessment as the second in order of importance.

Tolerability and safety data were calculated for all patients who were randomized to treatment and received at least one dose of blinded study medication. Adverse events are presented in frequency tables. The numbers of patients with "new" or "worsened" symptoms on the UKU scale (as compared with baseline) were compared between the two treatment groups by use of the Fisher exact test. All analyses were performed with SAS Version 6.12 or higher (SAS Institute, Cary, NC). 


\section{Results}

\section{PATIENT POPULATION}

One hundred seventy-eight subjects were screened to participate in the trial. Of these, 157 were randomly assigned to either treatment group and took at least one dose of study medication (mirtazapine, $\mathrm{N}=78$; venlafaxine, $\mathrm{N}=79$ ). Seventy-seven patients treated with mirtazapine and 75 with venlafaxine were included in the ITT group.

Both groups were well matched at baseline with respect to the demographic characteristics and mean group scores on the rating scales used in the study (Table 1).

TABLE 1. Demographic and disease characteristics at baseline ${ }^{a}$

\begin{tabular}{|c|c|c|}
\hline \multirow[b]{2}{*}{ Variable } & \multicolumn{2}{|c|}{ Group } \\
\hline & $\begin{array}{l}\text { Mirtazapine } \\
\quad(\mathrm{N}=78)\end{array}$ & $\begin{array}{l}\text { Venlafaxine } \\
(\mathrm{N}=79)\end{array}$ \\
\hline \multicolumn{3}{|l|}{ Gender } \\
\hline Male & $37.2 \%$ & $31.6 \%$ \\
\hline Female & $62.8 \%$ & $68.4 \%$ \\
\hline \multicolumn{3}{|l|}{ Age (yr) } \\
\hline Mean $\pm \mathrm{SD}$ & $45.9 \pm 10.4$ & $44.5 \pm 10.8$ \\
\hline Range & $25.4-69.3$ & $20.4-67.6$ \\
\hline Weight, $\mathrm{kg}($ mean $\pm \mathrm{SD})$ & $69.1 \pm 13.9$ & $67.6 \pm 17.4$ \\
\hline \multicolumn{3}{|c|}{ Previous episodes of major depression } \\
\hline \multicolumn{3}{|c|}{ Single episode } \\
\hline With hospitalization & $19.2 \%$ & $19.5 \%$ \\
\hline Without hospitalization & $30.8 \%$ & $20.5 \%$ \\
\hline \multicolumn{3}{|l|}{ Two or more episodes } \\
\hline With hospitalization & $33.3 \%$ & $28.6 \%$ \\
\hline Without hospitalization & $26.9 \%$ & $35.9 \%$ \\
\hline \multicolumn{3}{|c|}{ Duration of the present episode } \\
\hline 2 weeks to 1 month & $23.1 \%$ & $22.8 \%$ \\
\hline $1-6$ months & $67.9 \%$ & $65.8 \%$ \\
\hline 6-12 months & $9.0 \%$ & $11.4 \%$ \\
\hline HAM-D-17 & $29.5 \pm 3.0$ & $29.2 \pm 2.9$ \\
\hline MADRS & $34.6 \pm 5.3$ & $34.1 \pm 5.7$ \\
\hline CGI-Severity of Illness & $5.4 \pm 0.7$ & $5.4 \pm 0.7$ \\
\hline QLESQ & $27.4 \pm 7.4$ & $28.2 \pm 9.0$ \\
\hline QLDS & $24.6 \pm 7.4$ & $22.2 \pm 8.6$ \\
\hline UKU & $25.1 \pm 7.5$ & $21.3 \pm 8.4$ \\
\hline Concomitant medication & $96.2 \%$ & $93.7 \%$ \\
\hline \multicolumn{3}{|l|}{ Most frequent } \\
\hline Oxazepam & $56 \%$ & $49 \%$ \\
\hline Zolpidem & $35 \%$ & $41 \%$ \\
\hline
\end{tabular}

aHAM-D-17, 17-item Hamilton Rating Scale for Depression; MADRS, Montgomery-Åsberg Depression Rating Scale; CGI, Clinical Global Impression Scale; QLESQ, Quality of Life, Enjoyment and Satisfaction Questionnaire; QLDS, Quality of Life in Depression Scale; UKU, Utvalg for Kliniske Undersogelser side effect scale. 


\section{MEAN DAILY DOSES OF STUDY MEDICATION}

In the ITT population, the mean daily dose $( \pm S D)$ throughout the whole study period (including the 9-day rapid up-titration) was $49.5 \pm 8.3 \mathrm{mg} /$ day of mirtazapine and $255.0 \pm 59.8 \mathrm{mg} /$ day of venlafaxine.

\section{DROPOUTS}

More patients treated with mirtazapine (76.9\%) than venlafaxine (64.6\%) completed the study. Statistically significantly more patients treated with venlafaxine (15.3\%) than mirtazapine (5.1\%) dropped out from the study because of intolerable adverse events $(\mathrm{p}=0.037$; Table 2$)$.

TABLE 2. Number (\%) of dropouts in either treatment group (all randomized patients)

\begin{tabular}{lcc}
\hline & \multicolumn{2}{c}{ Group } \\
\cline { 2 - 3 } Reason & Mirtazapine & Venlafaxine \\
& $(\mathrm{N}=78)$ & $(\mathrm{N}=79)$ \\
\hline Adverse events & $4(5.1)$ & $12(15.3)^{a}$ \\
Subject not willing to continue & $7(9.0)$ & $10(12.7)$ \\
Other reasons & $7(9.0)$ & $6(7.6)$ \\
Total & $18(23.1)$ & $28(35.4)$ \\
\hline
\end{tabular}

$\mathrm{p}=0.037$, venlafaxine versus mirtazapine.

\section{EFFICACY}

The group mean MADRS and HAM-D-17 scores decreased from baseline at all assessment points in both treatment groups, indicating a substantial improvement in overall depressive symptoms. On the MADRS, although not statistically significantly different, the magnitudes of reductions were somewhat larger with mirtazapine at all assessment times. At week 4, the estimate of treatment difference of 2.3 points in favor of mirtazapine reached the level of clinical relevance16 (95\% confidence interval [CI]: -5.9 to $1.2 ; \mathrm{p}=0.195$ ) (Fig. 1). Similar results were seen on the HAM-D-17, with a somewhat larger reduction with mirtazapine at all assessments and a trend in favor of mirtazapine ${ }^{16}$ at week 4 (estimate of treatment difference, $-2.6 ; 95 \% \mathrm{CI}$, -5.1 to $0.0 ; \mathrm{p}=0.055$ ) and week 8 (estimate of treatment difference, $-2.1 ; 95 \% \mathrm{CI},-5.0$ to $0.8 ; \mathrm{p}=$ 0.154) (Fig. 2). Both treatments resulted in similar reductions from baseline in HAM-D anxiety and retardation factors at all assessment points. However, on the sleep disturbance factor, treatment with mirtazapine resulted in statistically significantly larger changes from baseline than treatment with venlafaxine from week 1 onward. This was maintained at all assessment times throughout the study (Table 3) (week 1, p = 0.022; weeks 2-8, p $\geq 0.001$ ).

In addition, treatment with mirtazapine resulted in higher percentages of responders and remitters. Although this difference was not statistically significant, at all assessment points during the 8-week treatment period, a higher percentage of responders and remitters was observed in the mirtazapine group than in the venlafaxine group, with both the MADRS and the HAM-D-17 criteria (Fig. 3). 
CGI Severity of Illness scores decreased in both treatment groups, indicating that the illness became less severe. In line with the results for primary efficacy variables, larger magnitudes of reduction were observed in the mirtazapine group (Table 3). Subjects in both groups became more satisfied with their quality of life, as assessed with both QLESQ and QLDS (Table 3).

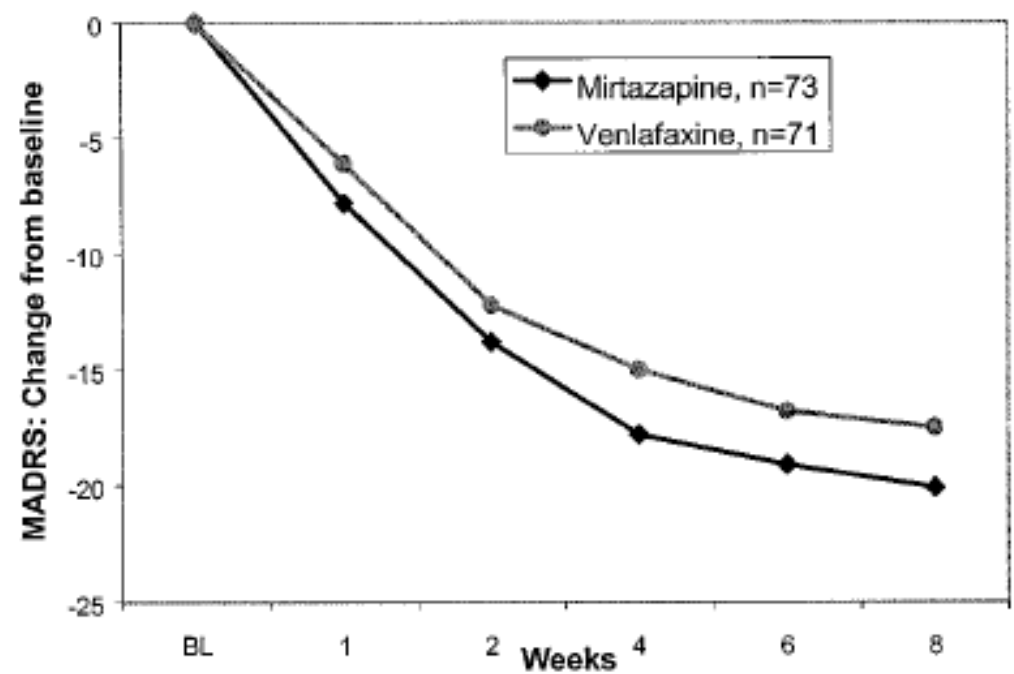

FIG. 1. Changes from baseline (BL) in mean group MADRS scores during 8 weeks of treatment with either mirtazapine (15-60 mg/day; $\mathrm{N}=73$ evaluable patients) or venlafaxine (75-375 mg/day; $\mathrm{N}=71$ evaluable patients). Baseline scores: mirtazapine group, 34.6; venlafaxine group, 33.9.

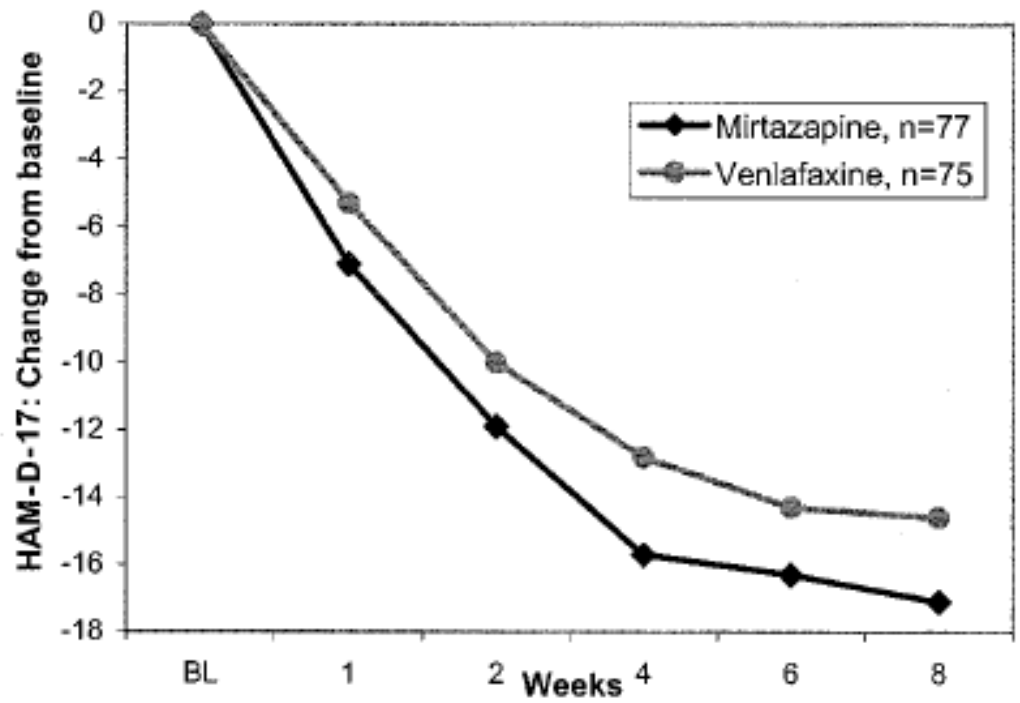

FIG. 2. Changes from baseline (BL) in mean group HAM-D-17 scores during 8 weeks of treatment with either mirtazapine (15-60 mg/day; $\mathrm{N}=77$ evaluable patients) or venlafaxine (75-375 mg/day; $\mathrm{N}=75$ evaluable patients). Baseline scores: mirtazapine group, 29.5; venlafaxine group, 28.9. 


\section{TOLERABILITY}

Slightly more patients treated with mirtazapine $(\mathrm{N}=58 ; 74.4 \%)$ than with venlafaxine $(\mathrm{N}=52$; $65.8 \%$ ) reported at least one adverse event (Table 4). Discontinuation rates resulting from intolerable adverse events have been described previously. One patient in the venlafaxine group committed suicide on study day 5 .

In general, incidences of reported adverse events were similar in both treatment groups. The observed differences between mirtazapine and venlafaxine were predictable (Table 5). In the mirtazapine group, the most frequently reported adverse events were complaints of weight increase (by $10.3 \%$ of patients), followed by dry mouth $(9.0 \%)$, headache (7.7\%), sleepiness (7.7\%), nausea (6.4\%), nervous tension (5.1\%), sedation (5.1\%), and accommodation disturbances (5.1\%). In the venlafaxine group, increased sweating was reported by $19.0 \%$ of patients, followed by constipation (15.2\%), headache (11.4\%), nausea (10.1\%), orthostatic hypotension (6.3\%), and decreased salivation (6.3\%).

For the following side effects described in the UKU scale, the incidences differed more than $10 \%$ between the mirtazapine and the venlafaxine group, respectively: nausea (15.8\% vs. $27.0 \%)$, constipation $(17.1 \%$ vs. $31.1 \%, \mathrm{p}=0.056)$, orthostatic dizziness $(14.5 \%$ vs. $27.7 \%)$, increased tendency to sweat (15.8\% vs. $35.1 \%, \mathrm{p} \leq 0.05)$, weight loss $(21.1 \%$ vs. $39.2 \%, \mathrm{p} \leq 0.05)$, and weight gain $(57.9 \%$ vs. $36.5 \%, \mathrm{p} \leq 0.05)$.

No clinically relevant changes in blood pressure, heart rate, or laboratory variables were registered during the study. In the mirtazapine group, endpoint change from baseline in the body weight was $2.0 \pm 3.7 \mathrm{~kg}$, and in the venlafaxine group it was $-0.5 \pm 2.9 \mathrm{~kg}$. This difference was statistically significant $(\mathrm{p}=0.001)$.

TABLE 3. Efficacy variables at endpoint: estimates of treatment differences, corresponding $95 \%$ confidence intervals, and $p$ values (ITT group, LOCF analysis) ${ }^{a}$

\begin{tabular}{|c|c|c|c|}
\hline $\begin{array}{l}\text { Efficacy } \\
\text { Variable }\end{array}$ & $\begin{array}{c}\text { Estimate of } \\
\text { Treatment Difference } \\
\text { (Mirtazapine Minus } \\
\text { Venlafaxine) }\end{array}$ & $95 \% \mathrm{CI}$ & $p$ Value \\
\hline HAM-D-17 total factors & -2.1 & -5.0 to 0.8 & 0.154 \\
\hline Anxiety/somatization & -0.5 & -1.6 to 0.6 & 0.381 \\
\hline Retardation & -0.5 & -1.6 to 0.5 & 0.328 \\
\hline Sleep disturbance & -1.0 & -1.6 to -0.5 & $0.001^{\circ}$ \\
\hline MADRS total & -1.9 & -5.9 to 2.0 & 0.336 \\
\hline CGI Severity of illness & -0.2 & -0.7 to 0.3 & 0.404 \\
\hline QLESQ & -3.0 & -11.0 to 4.9 & 0.459 \\
\hline QLDS & 2.6 & -2.1 to 7.3 & 0.289 \\
\hline
\end{tabular}

aITT, intent to treat; LOCF, last observation carried forward; CI, confidence interval; HAM-D-17, 17-item Hamilton Rating Scale for Depression; MADRS, Montgomery-Åsberg Depression Rating Scale; CGI, Clinical Global Impression; QLESQ, Quality of Life, Enjoyment, and Satisfaction Questionnaire; QLDS, Quality of Life in Depression Scale. bFavorable for mirtazapine. 

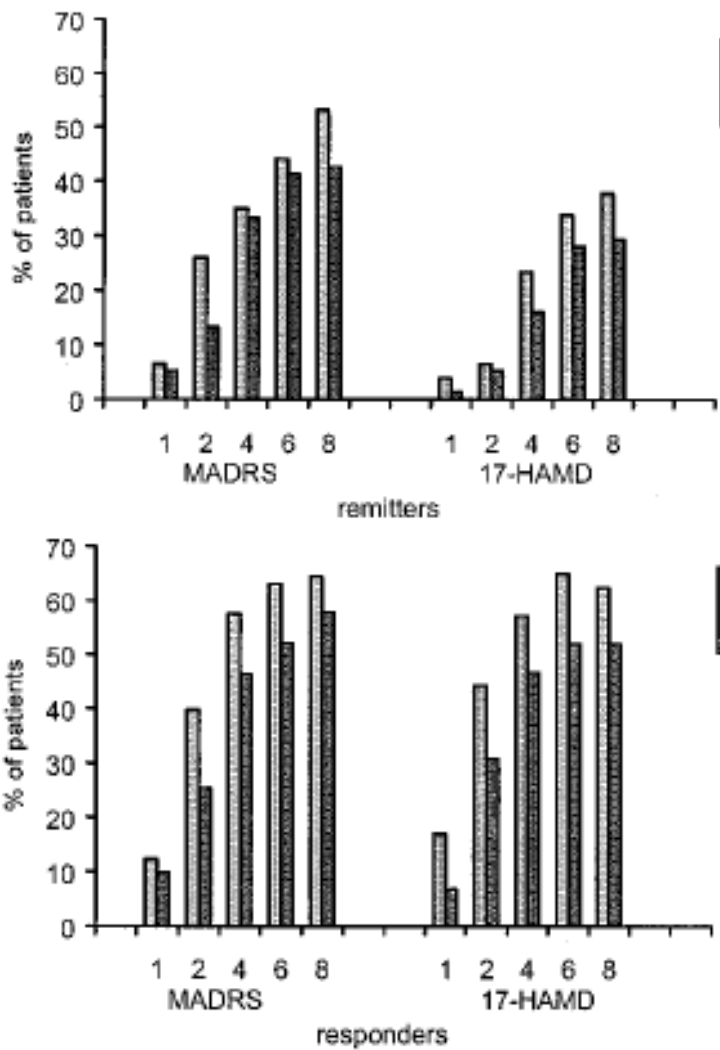

FIG. 3. Percentages of MADRS and HAM-D-17 responders (top) and remitters (bottom) as a function of time. Responders were defined as patients with at least a 50\% decrease from baseline scores. MADRS and HAM-D-17 remitters were defined as patients with a total score of $\leq 12$ and a total score of $\leq 7$, respectively.

TABLE 4. Percentage of adverse events reported in $\geq 5 \%$ of patients in either treatment group ${ }^{a}$

\begin{tabular}{lcc}
\hline & \multicolumn{2}{c}{ Group } \\
\cline { 2 - 3 } $\begin{array}{l}\text { Adverse Event } \\
\text { WHO Dictionary Term) }\end{array}$ & $\begin{array}{c}\text { Mirtazapine } \\
(\mathrm{N}=78)\end{array}$ & $\begin{array}{c}\text { Venlafaxine } \\
(\mathrm{N}=79)\end{array}$ \\
\hline Accommodation disturbances & 5.1 & 1.3 \\
Nervous tension & 5.1 & 5.1 \\
Sedation & 5.1 & 1.3 \\
Sleepiness & 7.7 & 5.1 \\
Dry mouth & 9.0 & 3.8 \\
Weight increase & 10.3 & 5.1 \\
Orthostatic hypotension & 1.3 & 6.3 \\
Decreased salivation & 1.3 & 6.3 \\
Nausea & 6.4 & 10.1 \\
Headache & 7.7 & 11.4 \\
Constipation & 3.8 & 15.2 \\
Increased sweating & 0.0 & 19.0 \\
\hline
\end{tabular}

aWHO, World Health Organization. 
TABLE 5. The UKU symptoms with an incidence of $\geq 10 \%$ in either treatment group, or a statistically significant difference between the groups ${ }^{a}$

\begin{tabular}{lcc}
\hline & \multicolumn{2}{c}{ Group } \\
\cline { 2 - 3 } & $\begin{array}{c}\text { Mirtazapine } \\
(\mathrm{N}=78)\end{array}$ & $\begin{array}{c}\text { Venlafaxine } \\
(\mathrm{N}=79)\end{array}$ \\
UKU Symptom & & \\
\hline Psychic side effects & 10.5 & 14.9 \\
Decreased duration of sleep & 13.2 & 10.8 \\
Failing memory & 16.4 & 10.8 \\
Asthenia & 19.7 & 16.2 \\
Increased duration of sleep & 19.7 & 18.9 \\
Emotional indifference & 19.7 & 18.9 \\
Tension & 21.1 & 16.2 \\
Sleepiness & 25.0 & 16.2 \\
Increased dream activity & & \\
Neurologic side effects & 11.8 & 8.1 \\
Dystonia & 14.5 & 9.5 \\
Paresthesias & 18.4 & 23.0 \\
Tremor & & \\
Autonomic side effects & $7.9^{b}$ & 0.0 \\
Increased salivation & 15.8 & 27.0 \\
Nausea & 9.2 & 1.4 \\
Diarrhea & 14.5 & 25.7 \\
Orthostatic dizziness & 15.8 & 14.9 \\
Accommodation disturbances & 15.8 & $35.1^{b}$ \\
Increased tendency to sweat & 17.1 & $31.1^{c}$ \\
Constipation & 21.1 & 29.7 \\
Reduced salivation & 22.4 & 24.3 \\
Palpitations & & \\
Other side effects & 3.9 & $13.5^{b}$ \\
Increased sexual desire & 10.7 & 9.1 \\
Ejaculatory dysfunction (males) & 11.8 & 10.8 \\
Orgastic dysfunction & 11.8 & 13.5 \\
Decreased sexual desire & 21.1 & $39.2^{b}$ \\
Weight loss & 30.0 & 32.4 \\
Headache & $57.9^{b}$ & 36.5 \\
Weight gain & & \\
\hline
\end{tabular}

aValues presented are percentages. UKU, Utvalg for Kliniske Undersogelser side effect scale. bp $\leq 0.05$ (Fisher exact test). cp $=0.056$ (Fisher exact test).

\section{Discussion}

This was the first study comparing the antidepressant efficacy and tolerability of mirtazapine and venlafaxine in hospitalized patients diagnosed with DSM-IV severe depressive episode with melancholic features. Both drugs were effective for improving patients' overall symptoms of depression and their quality of life. Although no statistically significant differences were registered between the treatments on primary efficacy measures, mirtazapine was consistently associated with somewhat larger magnitudes of change from baseline on both the HAM-D-17 and MADRS, and this difference was clinically relevant at week 4 (for both HAM-D-17 and MADRS) and endpoint (for HAM-D-17) (a difference of $\geq 2$ points on either HAM-D-17 or 
MADRS). ${ }^{16}$ In addition, also not statistically significant, there was a consistent advantage for mirtazapine in percentages of patients classified as responders and remitters, by use of both the HAM-D-17 and MADRS criteria. Mirtazapine produced statistically significant improvements from baseline in HAM-D sleep disturbance factor at all assessments.

In this study, the mean doses were $49.5 \pm 8.3 \mathrm{mg} /$ day for mirtazapine and $255.0 \pm 59.8 \mathrm{mg} /$ day for venlafaxine over the complete 8-week period, including the 9-day rapid up-titration. These high dosages were in line with the dosages used in similar trials with these kinds of patients. $2,3,5$

More patients treated with mirtazapine (76.9\%) than venlafaxine (64.6\%) completed the study. Although more of the patients treated with mirtazapine reported at least one adverse event compared with those treated with venlafaxine, statistically significantly more patients treated with venlafaxine $(15.3 \%)$ than with mirtazapine (5.1\%) dropped out because of intolerable adverse events.

In terms of reported adverse events and side effects registered on the UKU scale, the tolerability profiles of both drugs observed in this study did not differ from those previously reported. ${ }^{4,17}$

Studies in hospitalized patients with severe depression and melancholia are rarely conducted. There are data showing that patients with severe depression respond less well to antidepressant therapy than those with moderate depression, ${ }^{18}$ whereas melancholia was generally held to be responsive to antidepressant treatment. 19,20

Another drawback to studies with severe depression is that they do not always differentiate between complete or partial responders when baseline scores are high. Individuals who are severely depressed with a baseline HAM-D score of, e.g., 30 would still be symptomatic, even with a 50\% reduction, at the end of the study. 21 Nevertheless, a large majority of the currently available body of evidence regarding the efficacy of different classes of antidepressants in melancholic depression is derived from post hoc analyses. ${ }^{1}$ Among new antidepressants, venlafaxine has demonstrated its antidepressant efficacy over placebo in treatment of hospitalized patients with major depression and melancholia, ${ }^{2}$ whereas dual-acting antidepressants, such as the TCA clomipramine, ${ }^{22,} 23$ the SNRI venlafaxine, ${ }^{3}$ and the NaSSA mirtazapine, ${ }^{5-7}$ have been consistently shown to outperform single-acting SSRIs in this patient population.

In this patient population, which is characterized by poor sleep and early awakening, the statistically significant improvements in the HAM-D sleep disturbance factor with mirtazapine throughout the study may have important clinical implications. On the basis of the pharmacologic profile, a difference in sexual dysfunction in favor of mirtazapine was expected as well. However, the fact that the population in this study was hospitalized patients with severe melancholic depression may explain that no differences in sexual functioning between the two treatments were observed.

In conclusion, this study shows that both of the dualacting antidepressants mirtazapine and venlafaxine are effective for treating hospitalized patients with severe depression and melancholic features. Short-term treatment with mirtazapine seemed better tolerated, and this was demonstrated by the significantly higher dropout rate caused by adverse events in the venlafaxine group. 
Published in : Journal of Clinical Psychopharmacology (2001), vol. 21, n4, pp. 425-431 DOI:10.1097/00004714-200108000-00010

Status : Postprint (Author's version)

\section{Acknowledgment}

Supported by a clinical research grant from N.V. Organon, Oss, The Netherlands. 


\section{References}

1. Amsterdam JD. Selective serotonin reuptake inhibitor efficacy in severe and melancholic depression. J Psychopharmacol 1998;12[suppl B]:S99-S111.

2. Guelfi JD, White C, Hackett D, et al. Effectiveness of venlafaxine in patients hospitalized for major depression and melancholia. J Clin Psychiatry 1995;56:450-8.

3. Clerc GE, Ruimy P, Verdeau-Paillès J, et al. A double-blind comparison of venlafaxine and fluoxetine in patients hospitalized for major depression and melancholia. The Venlafaxine French Inpatient Study Group. Int Clin Psychopharmacol 1994;9:139-40.

4. Holm KJ, Markham A. Mirtazapine: a review of its use in major depression. Drugs 1999;57:607-31.

5. Wheatley DP, van Moffaert M, Timmerman L, et al. Mirtazapine: efficacy and tolerability in comparison with fluoxetine in patients with moderate to severe major depressive disorder. Mirtazapine-Fluoxetine Study Group. J Clin Psychiatry 1998;59:306-12.

6. Leinonen E, Skarstein J, Behnke K, et al. Efficacy and tolerability of mirtazapine versus citalopram: a double-blind, randomized study in patients with major depressive disorder. Nordic Antidepressant Study Group. Int Clin Psychopharmacol 1999;14:329-37.

7. Benkert O, Szegedi A, Kohnen R. Mirtazapine compared with paroxetine in major depression. J Clin Psychiatry 2000;61:656-63.

8. Hamilton M. A rating scale for depression. J Neurol Neurosurg Psychiatry 1960;23:56-62.

9. Montgomery SA, Åsberg M. A new depression rating scale designed to be sensitive to change. $\mathrm{Br} \mathrm{J}$ Psychiatry 1979;134:382-9.

10. Guy W. ECDEU assessment manual for psychopharmacology, revised. Department of Health, Education, and Welfare, Publication no. (ADM) 76-338. Rockville, MD: National Institutes of Mental Health, 1976:218-222.

11. Endicott J, Nee J, Harrison W, et al. Quality of Life, Enjoyment and Satisfaction Questionnaire: a new measure. Psychopharmacol Bull 1993;29:321-6.

12. Hunt SM, McKenna SP. The QLDS: a scale for the measurement of quality of life in depression. Health Policy 1992;22:307-19.

13. Lingjaerde 0 , Ahlfors UG, Bech $P$, et al. The UKU side effect rating scale. Acta Psychiatr Scand 1987;76[suppl 334]:1-100.

14. World Health Organization. Adverse reactions terminology (WHOART). Geneva: World Health Organization, 1997.

15. Koch GC, Carr J. Categorical data analysis. In: Berry DA, ed. Statistical methodology in pharmaceutical sciences. New York: Marcel Dekker, 1990:389-473.

16. Montgomery SA. Clinically relevant effect sizes in depression. Eur Neuropsychopharmacol 1994;4:283-4.

17. Dierick M. A review of the efficacy and tolerability of venlafaxine. Eur Psychiatry 1997;12[suppl 4):S307-S313. 
18. Kocsis JH, Croughan JL, Katz MM, et al. Response to treatment with antidepressants of patients with severe or moderate nonpsychotic depression and of patients with psychotic depression. Am J Psychiatry 1990;147:621-4.

19. Keller MB. Diagnostic issues and clinical course of unipolar illness. Review of psychiatry. Washington, DC: American Psychiatric Press, 1988;7:188-212.

20. Prien RF. Somatic treatment of unipolar depressive disorder. Review of psychiatry. Washington, DC: American Psychiatric Press, 1988;7:213-34.

21. Hirschfeld RMA. Efficacy of SSRIs and newer antidepressants in severe depression: comparison with TCAs. J Clin Psychiatry 1999;60:326-35.

22. Danish University Antidepressant Group. Citalopram: clinical effect profile in comparison with clomipramine. A controlled multicentre study. Psychopharmacology 1986;90:131-8.

23. Paroxetine: a selective serotonin reuptake inhibitor showing better tolerance, but weaker antidepressant effect than clomipramine in a controlled multicenter study. Danish University Antidepressant Group. J Affect Disord 1990;18:289-99. 